\title{
El perfil del alumno en el marco universitario de la enseñanza- aprendizaje
}

\begin{abstract}
Eva Domenech $^{a}$, Jorge Zamoranob, Marisol Juan-Borrás ${ }^{c}$, Isabel Escriche ${ }^{d}$
anstituto Universitario de Ingeniería de Alimentos para el Desarrollo. Departamento de Tecnología de alimentos. Universitat Politècnica de València. Camino de Vera, s/n, 46022 Valencia, Spain. evdoan@tal.upv.es, ${ }^{\text {b}}$ Escuela Técnica Superior de Ingeniería del Diseño. Universitat Politècnica de València. Camino de Vera, s/n, 46022 Valencia, Spain. jorzaes@etsii.upv.es, 'Instituto Universitario de Ingeniería de Alimentos para el Desarrollo. Departamento de Tecnología de alimentos. Universitat Politècnica de València. Camino de Vera, s/n, 46022 Valencia, Spain. majuabor@iad.upv.es, ${ }^{c}$ Instituto Universitario de Ingeniería de Alimentos para el Desarrollo. Departamento de Tecnología de alimentos. Universitat Politècnica de València. Camino de Vera, s/n, 46022 Valencia, Spain. iescrich@tal.upv.es.
\end{abstract}

\section{Resumen}

Los profesores universitarios, en el contexto de la enseñanza-aprendizaje, se están enfrentando a nuevos retos marcados por una sociedad educativa cada vez más influenciada por la globalización y los nuevos modelos pedagógicos. En este sentido, el objetivo del presente trabajo es conocer el perfil del alumno de la asignatura de Trazabilidad y Seguridad Alimentaria (enmarcada en el Máster profesionalizante de Ingeniería Agronómica de la $U P V)$, así como sus preferencias con relación al tipo de clase y a las actividades en grupo que se proponen durante el transcurso de la misma. Esta información ha sido obtenida mediante encuestas cumplimentadas por 65 alumnos. El 81.5\% de ellos son menores de 26 años, el 37\% compaginan estudios con trabajo, y aproximadamente el $65 \%$ ha realizado prácticas en empresa. En cuanto a la asignatura, el 70\% afirma poder llevar la materia al día y al $26 \%$ le gustaría el modelo de "clase inversa". En relación a la explicación de los contenidos teóricos por parte del profesor, el $61 \%$ prefiere que sea completa, frente a un $37 \%$ que valora más que sólo se haga un resumen. Los recursos más utilizados por el alumno para estudiar son una plataforma de gestión del aprendizaje (LMS) utilizado en la UPV, denominada "PoliformaT", "libros" y "videos". Los trabajos que se realizan en la asignatura son considerados muy útiles, aunque en el cómputo total del Máster, consideran tener excesivos trabajos. El 63\% prefiere que estos trabajos sean en grupo y un 56\% ven necesario una puntuación individual para reflejar la implicación que cada uno de ellos ha tenido. En 
conclusión, la información obtenida permite plantear algunas mejoras en la metodología de la asignatura para adaptarla a las preferencias de aprendizaje y opiniones de nuestros alumnos y de este modo conseguir un máximo rendimiento.

Palabras clave: Perfil de alumno, metodología docente, aprendizaje.

\section{Introducción}

El Espacio Europeo de Educación Superior ha supuesto importantes cambios en el proceso de enseñanza-aprendizaje para la adquisición de competencias transversales y específicas (Cid, 2008). El proceso de convergencia establece la necesidad de utilizar estrategias y metodologías activas para el aprendizaje con la finalidad de desarrollar en los estudiantes la capacidad del aprendizaje autónomo, cooperativo y continuo dentro de la sociedad del conocimiento, de la información y de las nuevas tecnologías (Caldeiro, 2014; Álvarez, 2015). Hoy en día, resulta evidente que el modelo de la clase magistral, en el que el docente hablaba y el estudiante copiaba no tiene cabida. Los nuevos enfoques pedagógicos buscan rescatar al alumno de la pasividad y convertirlo en un agente activo con participación en el aula que interactúe con el profesor y sus compañeros.

El diseño de una guía docente implica un proceso de toma de decisiones en relación, no sólo a los contenidos, sino también a las actividades y recursos que se deben llevar a cabo para consolidar los conocimientos.

Entre las nuevas propuestas de enseñanza-aprendizaje que se plantean se encuentra el método inverso o "Flipper", que brinda mayor autonomía a los estudiantes, además de favorecer la comunicación profesor-alumno y entre compañeros (Bergmann \& Sams, 2014; Blasco et al., 2016; Prieto, 2017; González et al., 2017). El trabajo en equipo y los casos prácticos son también estrategias de aprendizaje destinadas a adquirir información, analizarla, organizarla y comunicarla de forma coherente, logrando una mayor autonomía en los aprendizajes de los alumnos (Vázquez et al., 2017).

Por otra parte, a nivel universitario y más concretamente a nivel de Máster, los alumnos son cada vez más heterogéneos, tanto en edad, titulación, procedencia, experiencia previa en prácticas, etc. Esto hace que cada uno de ellos tenga unas necesidades distintas y por lo tanto, no existe un modelo pedagógico universal capaz de satisfacer a todos por igual.

En este contexto, el objetivo del presente trabajo es conocer el perfil del alumno de la asignatura de Gestión de la Seguridad y la Trazabilidad en la Industria Alimentaria 
(enmarcada en el Máster profesionalizante de Ingeniería Agronómica de la UPV), así como sus preferencias con relación a la dinámica de clase y a las actividades en grupo que en ella se proponen durante el transcurso de la misma. Todo ello con la finalidad de adaptar la metodología de la asignatura al estudiante y de este modo maximizar su rendimiento académico, mejorar sus habilidades, destrezas y competencias previstas en la guía docente.

\section{Metodología}

Se recogió la opinión de 65 alumnos de la asignatura de Gestión de la Seguridad y la Trazabilidad en la Industria Alimentaria (Máster profesionalizante de Ingeniería Agronómica de la Universitat Politècnica de València). La tabla 1.1 muestra las preguntas formuladas en la versión definitiva de la encuesta que cumplimentaron los alumnos. En el cuestionario se plantearon preguntas relacionadas con las preferencias por el tipo de clase y con las actividades en grupo que se proponen durante el transcurso de la asignatura. Mediante un "pre-test" 10 alumnos corroboraron previamente la comprensión de dichas preguntas.

El tratamiento estadístico de los datos se realizó con el programa Statgraphics Centurión XVI, aplicando un análisis de la varianza multifactorial, para determinar el nivel de significación de los resultados obtenidos. Los diagramas de caja-bigote, obtenidos permitieron comparar de forma sencilla las pautas de variabilidad existentes entre los distintos conjuntos de datos. En todos los casos se ha tomado como límite de la significatividad estadística una $\mathrm{p}<0.05$.

\section{Resultados}

\subsection{Resultados del pre-test}

El estudio del "pre-test" destinado a comprobar la comprensión de las preguntas iniciales puso en evidencia problemas en dos de ellas, la 2 y la 9. La Fig. 1 muestra cómo dichas preguntas habían sido formuladas con anterioridad a la versión definitiva del cuestionario que se ha mostrado en la tabla 1.1. La pregunta 2 se planteó para que los alumnos asignaran un porcentaje de 0 a 100 a cada recurso, según su utilidad para estudiar; sin embargo, se interpretaba mal ya que en vez de entender que cada recurso debía puntuarse individualmente, entendían que el conjunto de recursos debía sumar el 100\%. Para evitar esta confusión, se añadió la palabra "individualmente" y se separaron en filas cada uno de 
los recursos, evitando así que se vieran como un conjunto. Por otra parte, en la pregunta 9 algún entrevistado, al ser preguntado por el número de compañeros ideal de un grupo, pensó en el tamaño de la clase en lugar de los formados para realizar los trabajos y tareas propuestas por el profesor en el transcurso de la asignatura. En este caso, como se ve en la versión definitiva de la encuesta, tabla 1.1, se especificó "trabajo de clase" para evitar confusión.

Tabla 1.1. Preguntas formuladas en la encuesta

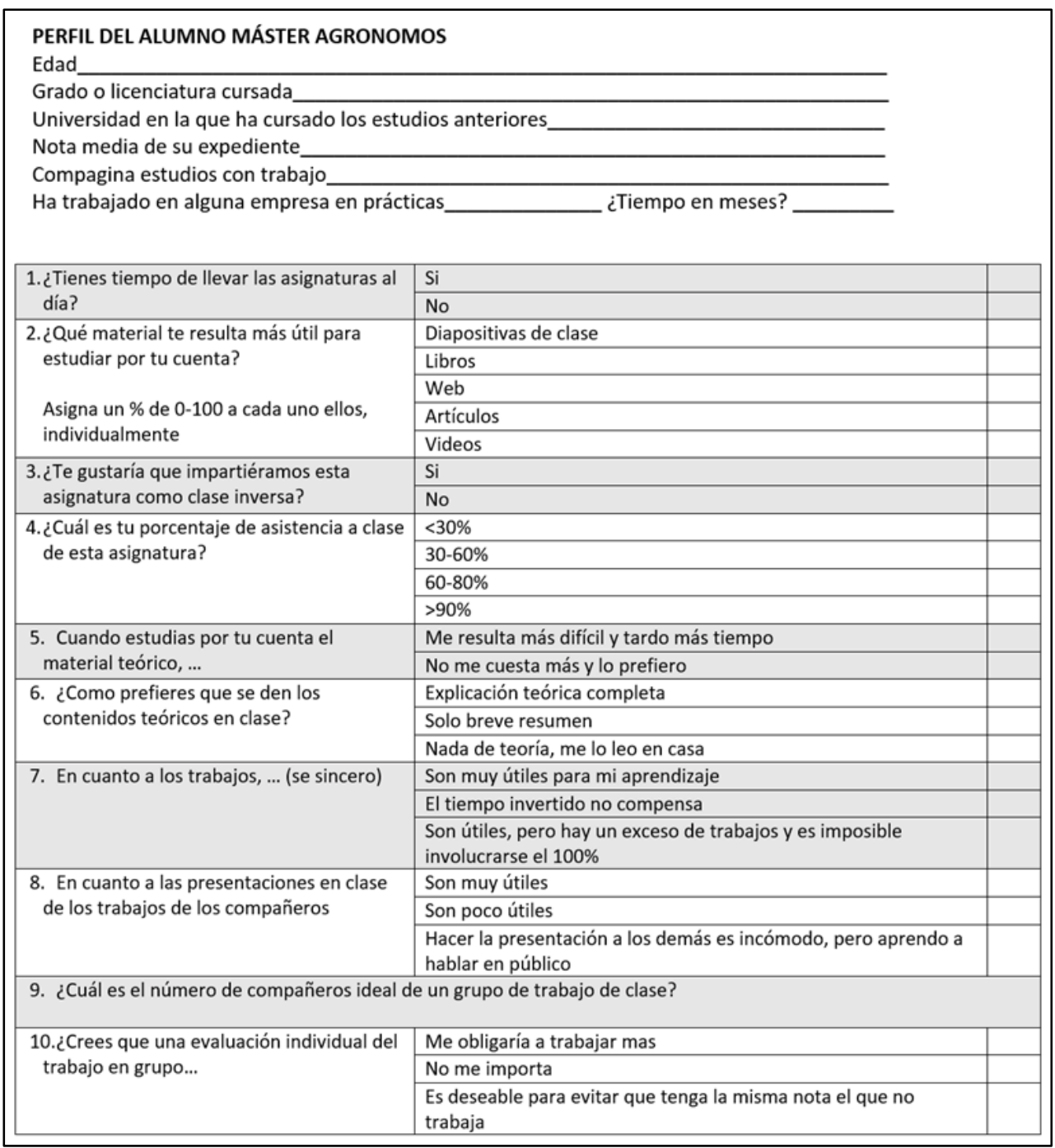

Fuente: Propia 


\begin{tabular}{|l|l|}
\hline $\begin{array}{l}\text { 2. ¿Qué te resulta más útil para estudiar por } \\
\text { tu cuenta? Puntúa del 1-100 cada recurso }\end{array}$ & $\begin{array}{l}\text { 9. ¿Cuál es el número de compañeros ideal } \\
\text { de un grupo? }\end{array}$ \\
$\begin{array}{l}\text { Diapositivas de clase__ } \\
\text { Libros } \\
\text { Web_ } \\
\text { Artículos_ } \\
\text { Videos__ }\end{array}$ & \\
\hline
\end{tabular}

Fig 1 Preguntas del pre-test antes de ser corregidas en la encuesta definitiva, presentada en la Tabla 1.1.

Fuente: Propia

\subsection{Perfil del alumno}

La tabla 1.2 muestra el perfil de los alumnos encuestados. El 81.5\% de ellos eran menores de 26 años, prácticamente todos provenían del grado de Ingeniería Agronómica (98.1\%), siendo el $75.9 \%$ de la UPV. En cuanto a su nota media de expediente, el $46.3 \%$ estaba por encima del 7.0. Sólo el $37 \%$ de ellos compaginaba estudios con trabajo y aproximadamente el 65\% había realizado prácticas en empresa antes de ingresar en el Máster.

Tabla 1.2. Perfil del alumno

\begin{tabular}{llcl}
\hline Características & Categoría & Respuestas $(\mathrm{n}=54)$ & Porcentaje \\
\hline Edad & $22-25$ & 44 & 81.5 \\
& $26-33$ & 7 & 13.0 \\
\multirow{4}{*}{ Grado/licenciatura } & Ingeniería agrónomos & 3 & 5.6 \\
& Ingeniero montes & 1 & 98.1 \\
Universidad & UPV & 41 & 1.9 \\
Nota expediente & No UPV España & 13 & 75.9 \\
& $5-7$ & 29 & 24.1 \\
Estudios y trabajo & $7.01-10$ & 25 & 53.7 \\
& Estudia y no trabaja & 34 & 46.3 \\
Prácticas en empresa & Prácticas realizadas sí & 20 & 63.0 \\
& Prácticas realizadas no & 19 & 37.0 \\
Tiempo prácticas & $1-3$ meses & 13 & 64.8 \\
& $4-6$ meses & 11 & 35.2 \\
& $7-12$ meses & 10 & 24.1 \\
& $>12$ meses & 1 & 20.4 \\
& & & 18.5 \\
\hline
\end{tabular}

Fuente: Propia 


\subsection{Opinión de los alumnos}

El $62 \%$ de los alumnos encuestados afirmaba poder llevar la asignatura al día. La Fig. 2. muestra de forma descriptiva mediante gráficos de cajas y bigotes los valores obtenidos en relaciónal tipo de material que resulta más útil para estudiar. Este gráfico permite la comparación de los diferentes conjuntos de datos, quedando el 50\% de los valores de cada grupo dentro de la caja. El recurso mejor valorado fue, con un valor medio del $66 \%$, las diapositivas de los temas impartidos en clase disponibles a través de la plataforma "PoliformaT" de la UPV. Es importante destacar que un 36\% de los estudiantes dieron a las diapositivas una importancia del 100\%. El segundo recurso más valorado fue los "libros" (27\%), al que el $14.5 \%$ de los alumnos le dio la máxima puntuación. En tercer lugar, se sitúan los "videos" con un valor medio del $25 \%$, pero solo el $7 \%$ le dio el máximo porcentaje. Entre los recursos menos valorados, con aproximadamente un $15 \%$ se encuentran "la web" y "los artículos". Hay que destacar que "la web" en ningún caso fue valorada con un $100 \%$ y sólo 3 de los 55 encuestados le dieron a "los artículos" la máxima puntuación.

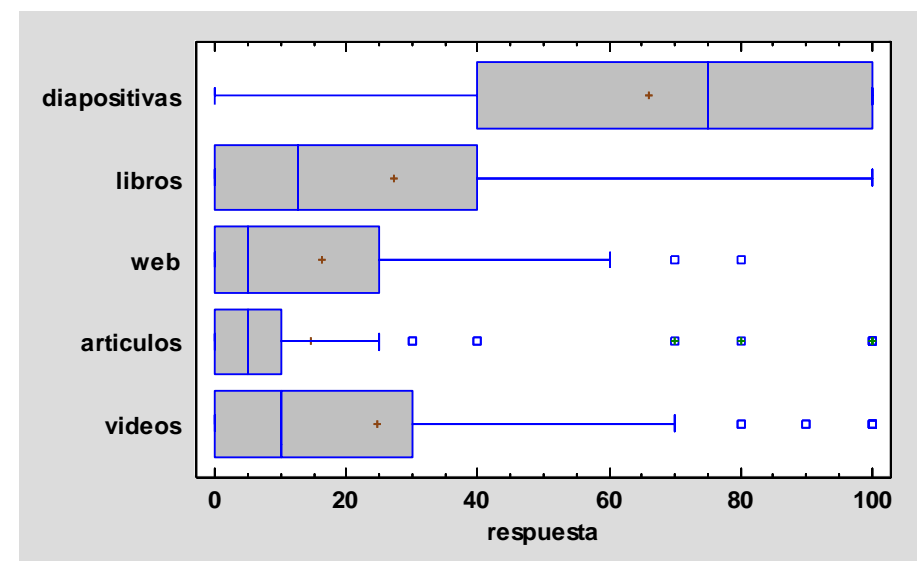

Fig. 2. Gráfico caja-bigotes sobre el uso de los distintos recursos didácticos Fuente: Propia

En relación a la clase inversa, sólo el $26 \%$ de los encuestados afirmó que le gustaría que la asignatura se impartiera de esta manera, sin diferencias significativas entre aquellos que compaginan trabajo/estudio y los que no (p valor 0.4110); si bien el número de respuestas en este sentido fue algo mayor entre los primeros.Tampoco se observaron diferencias atendiendo a la edad del encuestado ( $\mathrm{p}$ valor 0.6456), ni al porcentaje de asistencia a clase (p valor 0.2809). Este resultado concuerda con la afirmación de que estudiar el material teórico por su cuenta les resulta más difícil (92.5\%) y con la respuesta a la pregunta 
“Cómo te gustaría que se impartieran los contenidos teóricos en clase?”. En esta última, el $61.1 \%$ afirmó que completa, frente el $37.04 \%$ que prefería sólo un resumen y el $1.8 \%$ que no quería nada de teoría en clase. Por otra parte, el $100 \%$ de los que no querían nada de teoría y el $43.7 \%$ de los que preferían el resumen, son los que están más conformes a su vez con la clase inversa.

En cuanto a los trabajos en grupo, el $40 \%$ era partidario de hacerlos individualmente. Por otra parte, el $92.5 \%$ consideraba que son muy útiles, pero de ellos el $80 \%$ opinaba que hay un exceso de trabajos en el conjunto del Máster y es imposible involucrarse totalmente en todos. La mayoría de los alumnos (el 65\%) también opinaba que el tamaño de grupo más adecuado es "entre dos y cuatro compañeros". Con relación a la exposición, sólo el $25 \%$ afirmaba que es poco útil. Por último, en lo que respecta a la evaluación de los trabajos, el $55 \%$ pensaba que una valoración individual sería deseable para evitar compañeros que no participan; sin embargo, el $42.5 \%$ cree que no es necesario y un $2.5 \%$ afirmó que una evaluación individual le obligaría a trabajar más.

\section{Conclusión}

En el nuevo marco del proceso de enseñanza-aprendizaje, para la adquisición de competencias transversales y específicas, el alumno ha pasado a tener un papel protagonista y por lo tanto no puede excluirse en el momento de diseñar las metodologías a utilizar en la asignatura. De este modo, la encuesta propuesta en este trabajo aporta información útil para poder adaptarnos a las características del alumno que tenemos en clase. Así, conoceremos las necesidades de "nuestro público", permitiéndonos adaptar el modo de impartir la asignatura y de este modo aumentar la efectividad de nuestro trabajo.

Tomando como referencia los resultados obtenidos, los profesores de la asignatura darán en clase un breve pero completo resumen de los contenidos teóricos, que se complementarán con un libro editado para dicha asignatura y con el material didáctico disponible en la plataforma de gestión del aprendizaje, "PoliformaT". Los trabajos serán realizados por grupos de tres personas y se dará tiempo en las clases prácticas para realizarlo, con la finalidad de reducir la saturación de tareas a realizar en casa de las que se quejan los alumnos. Por último, habrá una evaluación individual por parte de los miembros de cada grupo, con relación a la participación de sus compañeros, que complementará la nota del profesor sobre el trabajo. De esta manera, se pretende motivar la participación de todos los miembros del grupo. 


\section{Referencias}

Álvarez, C. (2015). Teoría frente a práctica educativa: algunos problemas y propuestas de solución. Perfiles Educativos, 37(148), 172-190. https://doi.org/10.1016/j.pe.2015.11.014. http://www.sciencedirect.com/science/article/pii/S0185269815000264

Bergmann, J. \& Sams, A. (2014). Dale la vuelta a tu clase. Lleva tu clase a cada estudiante, en cualquier momento y cualquier lugar. Madrid: Ediciones SM.

Blasco, A. C., Lorenzo, J., Sarsa, J. (2016). La clase invertida y el uso de vídeos de software educativo en la formación inicial del profesorado. Estudio cualitativo. @tic. Revista d'innovació educativa, 17, 12-20.

Caldeiro, G. P. (2014). La comunicación en línea y el trabajo colaborativo mediado por tecnologías digitales. Austral Comunicación, 3(1), 13-30.

Cid, C. (2008). El uso de estrategias de aprendizaje y su correlación con la motivación de logro en estudiantes. Revista electrónica iberoamericana sobre calidad, eficacia y cambio en educación, 6(003), 100-120. https://repositorio.uam.es/handle/10486/661133

González, D., Jeong, J. S., Cañada, F. Gallego, A., (2017). La enseñanza de contenidos científicos a través de un modelo «Flipped»: Propuesta de instrucción para estudiantes del Grado de Educación Primaria. Enseñanza de las Ciencias, 35(2), 71-87. https://doi.org/10.5565/rev/ensciencias.2233

Prieto, A. (2017). Flipped Learning. Aplicar el modelo de aprendizaje inverso. Madrid: Narcea.

Vázquez, J., Hernández, J., Vázquez, J., Juárez, L. \& Guzmán, C. (2017). El trabajo colaborativo y la socioformación: un camino hacia el conocimiento complejo. Revista Educación y Humanismo, 19(33), 334-356. http:// dx.doi.org/10.17081/eduhum.19.33.2648 